\title{
Zur Faszinations- und Wissensgeschichte des Einhorns
}

\author{
Mensch-Tier-Beziehungen in historischer Perspektive
}

„Lebten Menschen mit Einhörnern zusammen?“ titelte das Online-Nachrichtenportal web. de am 1. April 2016. ${ }^{1}$ Die Meldung war indessen kein Aprilscherz, sondern rekurrierte auf aktuelle paläontologische Erkenntnisse: „Urzeitmenschen sind wahrscheinlich noch Einhörnern begegnet. Forscher haben in Kasachstan Überreste eines Sibirischen Einhorns gefunden, das noch vor rund 29.000 Jahren gelebt hat.“ Die Existenz von Einhörnern stellt diese Meldung in keiner Weise in Frage, wenn auch die beigegebene Illustration mit allen romantisierenden Vorstellungen gründlich aufräumt: Eine per Twitter-Meldung der California Academy of Sciences vom 28. März 2016 viral verbreitete Rekonstruktion ${ }^{2}$ zeigt, dass es sich bei den sibirischen Einhörnern nicht um anmutige und großäugige Wesen handelte, sondern vielmehr um „bisonartige[.] Urzeittiere mit Horn auf der Stirn“3. Das mag so manch eine zarte Seele erschüttert haben, doch bildet den eigentlichen Aufreger der sorglose und auf maximale Breitenwirkung zielende Umgang mit wissenschaftlichen Daten, der überhaupt zu dieser Meldung geführt hat.

In einer Ausgabe des Magazins der Süddeutschen Zeitung vom 20. Juli 2018 wurde unter dem Titel ,Das Scheinhorn' nachgezeichnet, wie der Meldung, Mensch und Einhorn hätten gleichzeitig (und das heißt: zeitgleich in derselben erdgeschichtlichen Periode und nicht etwa „zusammen“) gelebt, aufgrund einer fehlerhaften Datierung wissenschaftliche Evidenz zugesprochen werden konnte. Das in einer ersten Analyse ermittelte Alter eines einzelnen Knochenstücks von 28.000 Jahren wurde von dem Paläologen Andrej Schipanski ungeprüft veröffentlicht und erst später korrigiert: „Das gefundene Einhorn sei aber mindestens 50.000 Jahre alt. Einhorn und Mensch könnten sich demnach tatsächlich begegnet sein. Nur: Es lässt sich anhand des Knochens eben nicht bestimmen.“ ${ }^{4}$

1 http://web.de/magazine/wissen/lebten-menschen-einhoernern-31463742 (letzter Zugriff: 25.5.2021). Vgl. auch die Meldung ,Extinct 'Siberian unicorn' may have lived alongside humans, fossil suggests' in The Guardian vom 29.3.2016: https://www.theguardian.com/science/2016/mar/29/siberian-unicorn-extinct-humans-fossil-kazakhstan (letzter Zugriff: 25.5.2021).

2 https://twitter.com/calacademy/status/714510396372090880 (letzter Zugriff: 25.5.2021).

3 http://web.de/magazine/wissen/lebten-menschen-einhoernern-31463742 (letzter Zugriff: 25.5.2021).

4 Jan Schwenkenbecher, Das Scheinhorn, in: Süddeutsche Zeitung Magazin v. 20. Juli 2018.

> Das Werk ist unter der Creative-Commons-Lizenz Namensnennung - Weitergabe unter gleichen Bedingungen 4.0 International veröffentlicht. Den Vertragstext finden Sie unter: https://creativecommons.org/licenses/by-sa/4.0/deed.de. Bitte beachten Sie, dass einzelne, entsprechend gekennzeichnete Teile des Werks von der genannten Lizenz ausgenommen sein bzw. anderen urheberrechtlichen Bedingungen unterliegen können. 
Die Angelegenheit belegt eine beunruhigende Entwicklung in der wissenschaftlichen Betreuung von Zeitschriftenveröffentlichungen, denn der inkriminierte Artikel erschien im American Journal of Applied Science, das nach eigener Aussage „a variety of topics related to applied science ${ }^{\text {“5 }}$ publiziert. Die Zeitschrift gehört einer in Dubai angesiedelten Mediengruppe namens Science Publications an, die sich offenbar den zunehmenden Publikationsdruck kommerziell zunutze macht. In Bezug auf Einhörner aber werden zusätzlich zwei Dinge deutlich: Erstens ist die Faszinationskraft von Einhörnern immens, und zweitens unterliegt das Wissen von ihnen dem historischen Wandel. Jeder für sich genommen sind diese Befunde eher banal, doch eröffnen sich von hier aus Möglichkeiten, die Frage nach Mensch-Tier-Relationen historisch und in ihrer Historisierung dann auch didaktisch produktiv zu machen. Die Frage nach der Evidenz des Einhorns ist nämlich schon sehr viel früher als in der genannten spektakulären Meldung in Zusammenhang damit verhandelt worden, in welcher Beziehung Einhorn und Mensch zueinanderstehen. Unser Wissen vom Tier ist, das haben Arbeiten aus dem Kontext der ,Cultural and Literary Animal Studies' in den vergangenen Jahren gezeigt, ${ }^{6}$ in erheblichem Maße von menschlichen Projektionen bestimmt, und diese Voraussetzungen haben in historischer Perspektive nicht zuletzt auch die wissenschaftliche Auseinandersetzung mit Tieren geprägt. Tierwissen ist insbesondere in der Vormoderne kulturell geformt und wird in wirkmächtigen Erzählungen vermittelt. Der folgende Beitrag fragt anhand eines kleinen Ausschnitts aus der Faszinations- und Wissensgeschichte des Einhorns im europäischen Raum nach solchen Prozessen der Wissensproduktion und danach, wie sie die kollektive Vorstellung von Tieren bis in die Gegenwart hinein beeinflussen. ${ }^{7}$ Aus vormoderner Perspektive ist das Einhorn nämlich nicht, mit Rilke gesprochen, das „Tier, das es nicht giebt““ ${ }^{8}$ In der Antike und im Mittelalter gilt es nicht als Fabeltier, sondern man sucht sich ihm, wie auch die moderne Paläologie, mit den zur Verfügung stehenden naturkundlichen und hermeneutischen Methoden zu nähern. Es ging also die längste Zeit nicht um die Frage der Faktizität des Einhorns, sondern darum, wo man es findet, wie es aussieht, wie man es verwerten kann und wie man es fängt.

5 https://thescipub.com/announcement/2 (letzter Zugriff: 26.5.2021).

6 Vgl. methodisch zu den ,Cultural and Literary Animal Studies' Roland Borgards, Tiere in der Literatur. Eine methodische Standortbestimmung, in: Herwig Grimm/Carola Otterstedt (Hrsg.), Das Tier an sich. Disziplinenübergreifende Perspektiven für neue Wege im wissenschaftsbasierten Tierschutz. Göttingen 2012, 87-118, sowie zuletzt etwa die Beiträge in: Alexandra Böhm/ Jessica Ullrich (Hrsg.), Animal Encounters. Kontakt, Interaktion und Relationalität. Stuttgart 2019 (Cultural Animal Studies, Bd. 4.).

7 Der gewählte Ausschnitt kann notgedrungen nur klein bleiben, denn die vormoderne Auseinandersetzung mit dem Einhorn ist umfangreich. Das belegt die noch immer maßgebliche Studie von Jürgen W. Einhorn, die auf 521 Seiten Einhornwissen aus Antike und Mittelalter versammelt: Jürgen Werinhard Einhorn, Spiritalis Unicornis. Das Einhorn als Bedeutungsträger in Literatur und Kunst des Mittelalters. München 1976 (Münstersche Mittelalter-Schriften, Bd. 13.).

8 Rainer Maria Rilke, Die Sonette an Orpheus II.4, in: Ruth Sieber-Rilke (Hrsg.), Sämtliche Werke. Wiesbaden/Frankfurt a. M. 1955 (Rilke-Archiv, Bd. 1.), 753. 


\section{Das Einhorn in der Antike}

Bereits antike ethnographische Texte wie die Indika des Ktesias (um 398) enthalten Beschreibungen eines äußerst wilden gehörnten Tieres: „In Indien gibt es wilde Esel, die genau so groß sind oder sogar noch größer als Pferde. Ihre Körper sind weiß, ihre Köpfe dunkelrot, ihre Augen dunkelblau. Sie haben ein Horn von etwa anderthalb Fuß Länge auf der Stirn. Der Ansatz dieses Horns, der etwa zwei Handbreit über den Brauen liegt, ist reinweiß, der vordere Teil läuft in eine Spitze aus und ist von einem leuchtenden Purpurrot; der übrige Teil, das Mittelstück nämlich, ist schwarz. Wer aus einem dieser zu Trinkgefäßen verarbeiteten Hörner trinkt, leidet, wie es heißt, nicht mehr an Magenkrämpfen oder der heiligen Krankheit, Epilepsie. Er wird sogar immun gegenüber Giften [...]. Diese Tiere sind außerordentlich schnell und kraftvoll; keine anderen, weder ein Pferd noch irgendeins sonst, kann sie deswegen einholen. [...] Lebend lassen sie sich nicht einfangen. “9

Dieses Wissen wird, neben anderen antiken Autoren vermittelt über Aristoteles und Megasthenes, in die lateinischen Naturkunden übernommen. Auch Plinius der Ältere beschreibt in seiner Historia naturalis (wohl $77 \mathrm{n}$. Chr. beendet) ein in Indien vorkommendes, äußerst wildes Tier, „reliquo corpore equo similem, capite cervo, pedibus elephanto, cauda apro, mugitu gravi, uno cornu nigro media fronte cubitorum duum eminente. hanc feram vivam negant capi.“ - „das sonst am Körper dem Pferde, am Kopf aber dem Hirsch, an den Beinen dem Elefanten, am Schwanz dem Eber ähnlich ist, dumpf brüllt, während ein zwei Ellen langes schwarzes Horn mitten auf der Stirne hervorragt. Lebend soll sich dieses Wild nicht fangen lassen“" ${ }^{10}$

Im systematisierenden Zugriff der antiken Enzyklopädien erhält das Einhorn somit einen festen Platz in der Welt, die standardisierte Beschreibungsform erzeugt - in der Perspektive der mittelalterlichen Rezipient*innen - ihre eigene Evidenz. Der Fokus liegt dabei neben der Verwertbarkeit des Einhorns als Arzneimittel - ein Diskurs, auf den am Ende dieses Beitrags eingegangen wird - auf seiner Verfügbarkeit: Dieses Tier ist so wild, dass es sich nur schwer jagen lässt. Im Horizont des christlichen Mittelalters wird diese aus pagan-antiken Wissensquellen überlieferte Qualität des Einhorns durch den Befund ergänzt, dass die Übersetzer der griechischen Septuaginta die hebräische Bezeichnung ,re'em' (Auerochs oder Wildstier) in verschiedenen alttestamentlichen

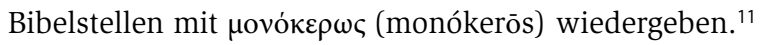

9 Zitiert nach: Chris Lavers, Das Einhorn. Natur, Mythos, Geschichte. Darmstadt 2010, 9f. Vgl. zu den Indika auch Einhorn, Spiritalis Unicornis (wie Anm. 7), 44.

10 C. Plinius Secundus d. Ä., Naturkunde. Lat.-dt. Buch VIII, hrsg. u. übers. v. Roderich König in Zus.arb. m. Gerhard Winkler. Kempten 1976, VIII.76, 64-65. Plinius differenziert zwischen ,monoceros' und ,rhinoceros', das er in VIII.71, 60-61 in Zusammenhang mit dem Triumph Pompeius' des Großen beschreibt.

11 Vgl. Bernd Roling, Der Wal als Schauobjekt: Thomas Bartholin (1616-1680), die dänische Nation und das Ende der Einhörner, in: Paul J. Smith/Karl A. E. Enenkel (Hrsg.), Zoology in Early Modern Culture. Intersections of Science, Theology, Philology and Political and Religious Education. Leiden 2014, 172-196, hier 172, Anm. 5; vgl. Einhorn, Spiritalis Unicornis (wie Anm. 7), 42-44. 
Interessant im Hinblick auf die Frage nach der Genese von Einhornwissen im christlichen Mittelalter sind dabei insbesondere diejenigen Stellen, die auf äußerst wilde

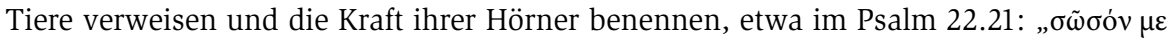

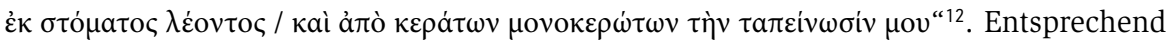
wandert das Tier als unicornis in die lateinische Übersetzung der „Vulgata“: „salva me ex ore leonis / et de cornibus unicornium exaudi me ${ }^{\text {“13. }}$. Martin Luther übersetzt dies 1545 mit „Hilff mir aus dem Rachen des Lewen / Vnd errette mich von den Einhörnern!“, während moderne Bibelübersetzungen die Psalmenstelle einhornfrei mit „Hörnern der Büffel“ (Elberfelder Bibel 1905; Einheitsübersetzung 2016) oder „Hörnern wilder Stiere“ (Lutherbibel 1984) bzw. „der wilden Stiere“ (Lutherbibel 2017) wiedergeben.

Den Bibelübersetzern und -kommentatoren der Spätantike stellte sich dabei ebenfalls nicht die Frage, ob es das Einhorn überhaupt „giebt“. Das Geschöpf ,re’em‘ stellte sie vielmehr vor das philologische Problem, ob es mit ,rhinoceros' oder ,monoceros ‘ korrekt wiederzugeben sei. Das wurde je nach Kontext unterschiedlich gelöst, ${ }^{14}$ doch wird in keinem der Fälle das antike Wissen vom Einhorn grundsätzlich in Frage gestellt. Damit gilt seine Existenz im Schöpfungsplan als abgesichert, was auch darin sichtbar wird, dass es zum festen Motivinventar bildlicher Darstellungen des Paradieses oder der Arche Noah gehört. ${ }^{15}$ Hier wird deutlich, in welchem Maße die Beziehungen von Menschen und Tier im christlichen Mittelalter vor dem Hintergrund der Schöpfungsgeschichte verhandelt werden: ${ }^{16}$ Die Paradieserzählungen entwerfen ein praelapsarisches Miteinander von Mensch und Tieren, das mit dem Sündenfall aufgekündigt wird. Der Pakt, den Noah nach der Sintflut mit Gott eingeht (Gen 9.1-5), ermächtigt den Menschen erneut und endgültig über die Schöpfung. Dieses Narrativ bildet überall dort den Verständnisgrund, wo die mittelalterliche Literatur Tiere thematisiert. Auffällig häufig werden etwa in der asketischen Erzählliteratur Begegnungen von Mensch und Tier an paradiesische Ursprünge rückgebunden, in denen „Wolf und Lamm beisammen weiden“ (Jes 65.25), wenn Tiere als Helfer in der Wüste oder Wildnis agieren. ${ }^{17}$ Zugleich verweisen Praktiken der Bemächtigung wie Jagd und Zähmung auf die durch den Lapsus eingezogene Zäsur in der Geschichte der MenschTier-Beziehungen. Immer wieder wird hier eine Faszination am unwiederbringlich

12 https://www.bibelwissenschaft.de/online-bibeln/septuaginta-lxx/lesen-im-bibeltext/bibel/text/lesen/stelle/19/210001/219999/ch/de02811fb5c811744e3d8b273fcfec0d/ (letzter Zugriff: 26.5.2021).

13 https://www.bibelwissenschaft.de/online-bibeln/biblia-sacra-vulgata/lesen-im-bibeltext/bibel/text/lesen/stelle/19/210001/219999/ch/d2f590559db148c23e5d4ef98b0d589b/ (letzter Zugriff: 26.5.2021).

14 Einhorn, Spiritalis Unicornis (wie Anm. 7), 48.

15 András Vizkelety, Einhorn, in: Engelbert Kirschbaum (Hrsg.), Lexikon der christlichen Ikonographie. Bd. 1, Freiburg 1968, 590-593, hier 593.

16 Udo Friedrich, Menschentier und Tiermensch. Diskurse der Grenzziehung und Grenzüberschreitung im Mittelalter. Göttingen 2009 (Historische Semantik, Bd. 5), 40-49.

17 Vgl. Julia Weitbrecht, Humanimale Heilsgemeinschaften. Intersektionale Perspektiven auf Mensch-Tier-Beziehungen in der Wüstenväterliteratur, in: Ingrid Bennewitz/Jutta Eming/Johannes Traulsen (Hrsg.), Gender Studies - Queer Studies - Intersektionalitätsforschung. Eine Zwischenbilanz aus mediävistischer Perspektive. Göttingen 2019 (Berliner Mediävistik- und Frühneuzeitforschung, Bd. 25), 417-437. 
verlorenen Naturzustand artikuliert, wird seine Wiederherstellung aus einer tiefen Sehnsucht heraus und zugleich im Wissen darum imaginiert, dass sich dieser Verlust nicht rückgängig machen lässt.

\section{Das Einhorn im Mittelalter}

Dieser Zusammenhang bildet - in einer spezifischen Genderkodierung - auch den Hintergrund der Faszinationsgeschichte des Einhorns in der christlich geprägten Vormoderne. Diese ,fera accerima', die „äußerst wilde Kreatur“, wird zwar als existent gedacht, bietet jedoch, anders als etwa Pferd oder Ziege, keinen alltagspragmatischen, funktionalen Anknüpfungspunkt: ${ }^{18}$ Das Einhorn ist ein Tier der Distanz und nicht der Nähe. Der imaginierte Zugriff auf das scheue Tier erfolgt in der christlichen Interpretation der antiken Naturkunden in der Tradition des Physiologus ${ }^{19}$ (der im 2. Jh. n. Chr. in Alexandria entstand und im Mittelalter in alle Volkssprachen übertragen wurde) in einer besonders exklusiven Form, welche die Wahrnehmung des Einhorns bis in die Gegenwart nachhaltig prägt. Dabei werden in der allegorischen Auslegung der antiken Natur,tatsachen ' die pagan-antiken und christlichen Wissensbestände zu einem wirkmächtigen Narrativ verbunden.

Auch der Physiologus folgt der naturkundlichen Beschreibungstradition der antiken Prätexte und benennt, nach einem einleitenden Verweis auf eine Psalmenstelle (Ps 92.11) die Eigenschaften des Einhorns: „Der Physiologus sagte vom Einhorn, dass es folgende Eigenheit habe: Es ist ein kleines Tier, ähnlich einem Böcklein, ist aber sehr hitzig: ein Jäger kann sich ihm nicht nähern, weil es sehr stark ist; es hat aber ein Horn mitten auf seinem Kopf. Wie wird es nun gefangen? Eine reine, schön gekleidete Jungfrau setzen sie vor ihm nieder, und es springt ihr auf den Schoß, und die Jungfrau nährt das Tier und bringt es dem König in den Palast. “20

Man hat versucht, diese eher ungewöhnliche Jagdtechnik auf die indische Mythologie zurückführen: „Einhorn“ heißt dort der Sohn eines Menschen und einer Gazelle, der ein einzelnes Horn auf der Stirn trägt und als Asket im Wald lebt. Er wird von einer schönen Frau aus seiner Eremitage gelockt, um dem unter einer Dürre leidenden Land in der Vereinigung der beiden Liebenden den Regen zu bringen. ${ }^{21}$ Auch hier wird über

\footnotetext{
18 Auch die Aussagen zur medizinischen Verwertbarkeit des kostbaren Horns verweisen eher auf seine Auratisierung. Zur Konjunktur als „frühe Form des Aspirin“ des Nordens kommt es erst in der Frühen Neuzeit, vgl. Roling (Anm. 11), 185.

19 Vgl. F. N. M. Diekstra, The ,Physiologus', the Bestiaries and Medieval Animal Lore, in: Neophilologus 69, 1985, 142-155; Nikolaus Henkel, Studien zum Physiologus im Mittelalter. Tübingen 1976 (Hermaea N.F., Bd. 38). Eine Fallstudie zum produktiven Tierwissen in mittelalterlichen Naturkunden bietet Anna-Theresa Kölczer, (Re-)Produktiver Physiologus. Zur Genese von Fuchswissen im Buch der Natur Konrads von Megenberg, in: Anna Chalupa-Albrecht/Maximilian Wick (Hrsg.), Wo die Epigonen wohnen. Epigonalität in mediävistischer Perspektive. Frankfurt a. M. 2020 (Mikrokosmos, Bd. 86), 293-325.

20 Physiologus. Griech./Dt., hrsg. u. übers. v. Otto Schönberger. Stuttgart 2001 (Reclams Universal-Bibliothek, Bd. 18124 ), 39.

21 Einhorn, Spiritalis Unicornis (wie Anm. 7), 35.
} 
das gehörnte Mischwesen die Grenze von Natur und Kultur verhandelt. Direkte Bezüge zu diesem Mythos sind für das christliche Mittelalter indessen nicht belegbar (auch handelt es sich bei der Frau in einigen Überlieferungen um eine Hetäre, die der Eremit Einhorn überdies für einen jungen Asketen hält, so dass die Begehrensstrukturen hier etwas unübersichtlich erscheinen). Anfänge im Sinne fixierten Wissens, wie die Jungfrau zum Einhorn kam, lassen sich nicht rekonstruieren, man kann lediglich die außerordentliche Produktivität dieses Narrativs in der christlichen Vormoderne zur Kenntnis nehmen. Mit der Ausbildung des Motivzusammenhanges von Jagd, Jungfräulichkeit und Zähmung im Physiologus erfolgt im Anschluss an die Beschreibung des Einhorns zugleich seine Auslegung, in der es „als Sinnbild unseres Erlösers“22, die jungfräuliche Lockspeise als Maria und die Zähmung als die Inkarnation Christi gedeutet wird. ${ }^{23}$

Mit dieser Integration naturkundlicher Wissensbestände in die christliche Deutungstradition des lateinischen Westens werden diese zugleich transformiert, woraus sich fortwährend etwas Neues eröffnet: So bildet sich in Prozessen umfänglicher Allegorisierung im Spätmittelalter etwa der Bildtypus der ,sakralen Einhornjagd ' aus, in der die Einhornjagd die Empfängnis Mariä versinnbildlicht. Die Jagd wird mit einer Fülle von Paradies- und Reinheitstopoi angereichert und in einen umfassenden heilsgeschichtlichen Kontext gestellt, innerhalb dessen sie zum Selbstopfer umgedeutet wird. ${ }^{24}$ Die Identifikation des Einhorns mit Christus ermöglicht daneben auch eine folgenreiche Übertragung im Kontext höfischer Liebeskonzeptionen: Das wilde und unzähmbare Einhorn versinnbildlicht hier den Mann - nicht nur im Sinne des Erlösers, sondern auch als Objekt der Begierde; die Jagd erscheint als Liebesjagd. ${ }^{25}$

Im Zuge dieser vielfältigen Resemantisierungen bleibt das Narrativ von Einhorn, Jungfrau und Zähmung stabil, doch verschiebt sich in Zusammenhang mit der durch die christliche Interpretation eingebrachten Reinheitsthematik immer wieder der Fokus vom scheuen Tier hin zu denen, die es fangen wollen. Auch in den höfischen Entwürfen wird am Einhorn weniger das Tier selbst verhandelt als vielmehr, wie es sich zum Menschen verhält und der Mensch sich in ihm spiegelt. Das verweist auf eine weitere Aktualisierung in der Geschichte vormoderner Mensch-Tier-Beziehungen, denn die höfische Erzählliteratur arbeitet sich ebenfalls am Verlust paradiesischer Ursprünglichkeit ab, entwirft die Annäherungen an die Natur aber in forcierter Künstlichkeit, als Szenarien übersteigerter Kultiviertheit, durch die man mit den gejagten, gezähmten und

22 Physiologus (wie Anm. 20), 39.

23 Zur Auslegungsgeschichte vgl. Einhorn, Spiritalis Unicornis (wie Anm. 7), 50-60.

24 Julia Weitbrecht, ,Thou Hast Heard Me from the Horns of the Unicorns'. The Biblical Unicorn in Late Medieval Religious Interpretation, in: Interfaces: A Journal of Medieval European Literature 5, 2018, 49-64, https://doi.org/10.13130/interfaces-05-05.

25 Vgl. zur Tradition der Liebesbestiarien Hans-Jürgen Scheuer, Aspekte einer vormodernen Poetik der animalia. Tierkataloge und Minnebestiare in mittelhochdeutscher Dichtung, in: Ders./Ulrike Vedder (Hrsg.), Tier im Text. Exemplarität und Allegorizität literarischer Lebewesen. Bern u. a. 2015, 37-60. 
dressierten Tieren zugleich auch die eigene Animalität zu bändigen sucht. ${ }^{26}$ Anders als in den asketischen Szenarien, die in der friedlichen Koexistenz von Mensch und Tier den paradiesischen Heilszustand zu reaktualisieren suchen, wird die Darstellung von Wildheit hier zu einer Problematisierung der höfischen Disposition genutzt. In einer Übertragung ins Weltliche ${ }^{27}$ wird der Aspekt der Bezähmung in den Affekthaushalt des Menschen verlagert, so dass der Zusammenhang von Jagd, Begehren und Keuschheit eine neue Perspektivierung erfährt. Dies erlaubt - noch vor der wissenschaftlichen Abwicklung des Einhorns zu Beginn der Frühen Neuzeit - auch ironische Distanzierungen, die jedoch (noch) nicht am faktischen Wissen über das Einhorn ansetzen, sondern an der Frage, ob es dem Menschen überhaupt möglich ist, das scheue Tier zu fangen.

In Rudolfs von Ems Weltchronik (Mitte des 13. Jahrhunderts) etwa wird das Einhorn zunächst gemäß der naturkundlichen Tradition als ein äußerst wildes Mischwesen beschrieben: „Da [in Indien] sint ouh einhúrnin: / den in der welte nieman / mit mannis kraft betwingin kann, / so starch ist er und alse balt. / sin lip ist alse ein ros gestalt. / hirzis houbit hat er vor, / das treit er vientlich embor. / sine site sint unsuoze. / er treit helfandis fuoze. / er ist gezagil als ein swin. / emmiten an der stirnin sin / hat er ein horn reht als ein glas, / vier fuoze lanc, als ich ez las: / vor deme kann sih niht irwern / noh mit dekeinir wer genern: / alse úbil ist das selbe tier, / so starch, so zuornic und so fier / ist ez und also unverzaget / das es niht wand ein reinú magit / gevahin mag [...]. “28 ([In Indien] kommt auch das Einhorn vor, das niemand auf der Welt mit Manneskraft bezwingen kann, so stark und schnell ist es. Sein Körper ist der eines Pferdes, es trägt sein Hirschhaupt auf feindselige Weise erhoben. Sein Verhalten ist unfreundlich. Es hat Elefantenfüße und einen Schweineschwanz. Mitten auf der Stirn trägt es ein Horn wie aus Glas, vier Fuß lang, habe ich gelesen. Vor ihm kann man sich nicht schützen und sich ihm mit keiner Waffe nähern, so böse ist dieses Tier, so stark, so zornig und so stolz ist es, und so furchtlos, dass niemand außer einer reinen Jungfrau es zu fangen vermag.)

Während die ungewöhnliche Jagdmethode in den naturkundlichen Texten lediglich benannt und dann allegorisch ausgelegt wird, verdeutlicht Rudolf zudem, unter welchen Umständen diese Jagd dann eben auch nicht gelingen kann: „Ist abir das si ist ein wip / und megde namin ir selbin giht, / so lat er si genesin niht / und zeigit an ir grozin zorn: / durh sie so stichet er das horn / und richet an ir die valscheit / die si von ir selber seit." ${ }^{\text {"29 }}$

26 Vgl. zu diesem Zusammenhang in Gottfrieds Tristan Julia Weitbrecht, On Courtly Discipline. Animal Rituals and Noble Self-fashioning in Medieval Courtly Literature, in: Mark Hengerer/Nasir Weber (Hrsg.), Animals and Courts. Europe, c. 12001800. Berlin/Boston 2019, 95-109.

27 Das könnte man mit Susanne Köbele und Bruno Quast als literarische Säkularisierung bezeichnen, vgl. die Beiträge in Susanne Köbele/Bruno Quast (Hrsg.), Literarische Säkularisierung im Mittelalter. Berlin 2014 (Beiträge zu einer kulturwissenschaftlichen Mediävistik, Bd. 4). Als, Entmythologisierung' der allegorischen Tradition versteht dagegen Jürgen W. Einhorn die Jagdszene im Wilhelm von Österreich, aus seiner motivgeschichtlichen Perspektive stellt dies eine Schwundstufe dar, denn „die Hochschätzung der Jungfräulichkeit bleibt erhalten, aber dem Tier werden keine edlen Gedanken mehr zugetraut;" Einhorn, Spiritalis Unicornis (wie Anm. 7), 165. In dieser Fokussierung auf die geistliche Perspektive wird jedoch die Produktivität des Motivzusammenhanges in der höfischen Literatur ganz ausgeblendet.

28 Rudolfs von Ems, Weltchronik, hrsg. v. Gustav Ehrismann. Berlin 1915 (Deutsche Texte des Mittelalters, Bd. 20), V. $1767-1786$.

29 Ebd., V. 1793-1799. 
(Wenn es sich aber so verhält, dass sie eine Frau ist und sich selbst als Jungfrau bezeichnet, dann lässt es sie nicht davonkommen / und beweist ihr gegenüber großen Zorn: Es durchbohrt sie mit seinem Horn und übt an ihr Vergeltung für die Lüge, die sie in Bezug auf sich selbst geübt hat.). Die Einhornjagd wird hier zur Jungfrauenprobe und erfährt eine gewisse Rationalisierung - ohne dass dabei die Existenz des Tieres und seiner Rolle darin in Frage gestellt würden.

Auch im Wilhelm von Österreich Johanns von Würzburg (vor 1314 entstanden) wird traditionelles Einhornwissen aufgerufen und im Rahmen eines Liebes- und Abenteuerromans narrativiert. Darin berichtet der Jägermeister der Hofgesellschaft um den Protagonisten Wilhelm, er habe Spuren eines Einhorns gefunden. Er ruft alle verfügbaren Wissensbestände zum Einhorn auf und weckt damit umgehend Wilhelms Begehren danach, „den nu [zu] gejagen, daz ich auch mo̊ht gesagen, daz ich ir ain het gesehen!“30 (es sofort zu jagen, damit ich auch sagen kann, ich hätte eines davon gesehen!) Als Lockmittel soll die Zofe von Aglaye, der Ehefrau Wilhelms, dienen. Diese äußert als einzige Skepsis an dieser Unternehmung: „Agly daz wiplich bilde / hazzt das gejægde; / si sprach zu der mægde: / ,du bist ein to̊rinne. / war hastu dine sinne /getan? Wiltu ain tier dich / lan ertoten daz sich / niht verstat umm sache kain? / und wærstu aller mægde rain, / zwar, ez nimpt dir den lip. ${ }^{31}$ (Aglaye, die schöne Frau, hasste diese Jagdunternehmung. Sie sprach zu der Jungfrau: ,Du bist eine Törin. Bist Du von Sinnen? Willst du dich von einem Tier töten lassen, das überhaupt keinen Verstand besitzt? Und wenn du die reinste aller Jungfrauen wärst, nimmt es dir wahrhaftig doch das Leben.').

Hier wird ebenfalls eine gewisse Rationalisierung des Wissens vom Einhorn artikuliert, doch richtet sie sich nicht auf die Qualität der menschlichen Lockspeise, sondern stellt vielmehr die Zähmbarkeit wilder Geschöpfe grundsätzlich in Frage. ${ }^{32}$ Dies ist sicherlich in kritischer Weise auf den fragilen Affekthaushalt einer Hofgesellschaft gerichtet, die sich von der Neugier, einmal ein echtes Einhorn zu sehen, in den wilden Wald locken lässt. Diese Jagd nimmt auch sogleich ein böses Ende: Das scheue Wesen wird nicht gefangen, ja nicht einmal erspäht; stattdessen wird die Jagdgesellschaft von einer Horde heranreitender Sarazenen abgelenkt, was den tragischen Tod von Wilhelm und Aglaye zur Folge haben wird. Das Ende des fragmentarisch erhaltenen Romans kennen wir nicht. Vom Einhorn bleiben nur die Spur und das Begehren, das es weckt - dieses Distanzwesen entzieht sich seinen menschlichen Jägern immer wieder aufs Neue und führt sie in die Irre, was die höfische Literatur anspielungsreich inszeniert.

30 Johann von Würzburg, Wilhelm von Österreich. Gothaer Handschrift, hrsg. v. Ernst Regel. Berlin 1906 (Deutsche Texte des Mittelalters, Bd. 3), V. 18863-18865.

31 Ebd., V. 18886-18895.

32 Vgl. zur Wildheit des Protagonisten sowie zur wildekeit als Erzählprogramm und poetisches Prinzip Sandra Linden, wildiu rede und ethische Funktion. Zum Konzept der wildekeit im ,Wilhelm von Ôsterreich' Johanns von Würzburg, in: Wolfram-Studien 25, 2018, 135-156. 
Nur weil die Jagdtechnik hinterfragt wird und das Beutetier in den Hintergrund tritt, verliert das Einhorn also noch lange nicht seinen Faktizitätsanspruch. Daher sind diese Beispiele aus der höfischen Literatur des hohen Mittelalters ebenfalls Bestandteil einer Wissensgeschichte vom Einhorn, denn sie beruhen auf der naturkundlichen Tradition, die ihm Evidenz zuspricht. Zugleich verdeutlichen die Texte, dass sich hier ein literarisches Wissen um die Dynamiken der Einhornjagd herausbildet, das auch eine gewisse ironische Distanz zum scheuen Objekt der Begierde ermöglicht. Ob man das Einhorn tatsächlich finden kann, spielt dabei gegenüber dem Begehren, das die menschlichen Jägerinnen und Jägern antreibt, eine untergeordnete Rolle - nicht selten erweist sich das Jagdobjekt nachträglich ohnehin nur als ein „vremeder hirz“, ein ,wundersamer Hirsch` wie im Tristan Gottfrieds von Straßburg. ${ }^{33}$

\section{Das Einhorn an der Schwelle zur Moderne}

Erst in der Frühen Neuzeit wird eine allmähliche Skepsis gegenüber der physischen Existenz des Einhorns fassbar. Das steht in Zusammenhang mit Schriften, die weiterhin auf die naturkundlichen Wissensbestände zurückgreifen, diese aber auch zunehmend kritisch überprüfen. Zunächst bilden sich Ansätze zur Empirie heraus, was paradoxerweise aber erst einmal gerade nicht zur Abschaffung des Einhorns führt: So macht etwa der Bericht Bernhard Breydenbachs von seiner Pilgerreise durch das Heilige Land im Jahr 1483/4 (gedruckt 1486) deutlich, in welchem Maße das aus der Bibel wie aus den antiken Naturkunden bekannte Wissen die empirische Naturbeobachtung prägen und Einfluss auf die Wirklichkeitserfahrung nehmen: „Jtem da selbet sahen wir eyn groß thyer was vil grosser dan eyn kemmel thyer / vnnd saget vns vnser gleytzman daz eß warlich were eyn eynhornn. - Item sahen wir dort ein großes Tier, viel größer als ein Kamel. Unser Führer sagte uns, dies sei wahrhaftig ein Einhorn.“34

Auch Breydenbachs zeitweiliger Reisegefährte Felix Fabri schildert in seinem lateinischen Bericht aus dem Heiligen Land diese Begegnung und beschreibt das überaus edle und einzigartige Tier, die „nobilissima“ und „singularissima bestia“"35, etwas genauer, wobei er sich auf die naturkundlichen Traditionen beruft. Das Tier habe „equino corpore, elephantinis pedibus, cauda suilla, buxei coloris“, also einen „Pferdeleib, Elefantenfüße,

33 Johannes Rathofer, Der, wunderbare Hirsch' der Minnegrotte, in: Alois Wolf (Hrsg.), Gottfried von Straßburg. Darmstadt 1973 (Wege der Forschung, Bd. 320), 371-391 (erstmals in: Zeitschrift für deutsches Altertum und deutsche Literatur 95, 1966, 27-42).

34 Bernhard von Breydenbach, Peregrinatio in terram sanctam: Eine Pilgerreise ins Heilige Land. Frühneuhochdeutscher Text und Übersetzung, hrsg. v. Isolde Mozer. Berlin/Boston 2010, fol. 139v, 550/551 (hier wie auch in den folgenden Zitaten wurde die Schreibweise leicht modernisiert). Vgl. Andreas Klussmann, In Gottes Namen fahren wir. Die spätmittelalterlichen Pilgerberichte von Felix Fabri, Bernhard von Breydenbach und Konrad Grünemberg im Vergleich. Saarbrücken 2012, 98.

35 Fratris Felicis Fabri, Evagatorium in Terrae Sanctae, Arabiae et Aegypti, hrsg. v. Konrad Dieter Hassler, Bd. 2. Stuttgart 1843 (Bibliothek des Literarischen Vereins in Stuttgart, Bd. 3), 441. 
einen Schweineschwanz und sei schiefergrau“36. Fabri weist auch auf die ihm aus den

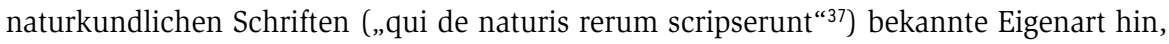
dass man dieses Tier nur mit Hilfe einer Jungfrau fangen könne. Die Passage schließt mit Verweisen auf die Erwähnungen des Einhorns in der Bibel und bindet somit das auf der Reise Erlebte zweifach an die traditionellen Wissensbestände zurück.

Eine entsprechende Gleichzeitigkeit der Orientierung an autoritativem Buchwissen und empirischer Betrachtung weist auch die Historia Animalium des Zürcher Arztes Conrad Gesner auf (1551-1558 entstanden und unter dem Titel Thierbuoch 1563 auch auf Deutsch erschienen ${ }^{38}$ ). Gesner versammelt alle ihm bekannten „alter und neuer Scribenten Meynungen“"39 zum Einhorn und befragt sie durchaus kritisch auf ihren Wahrheitsgehalt. Unter der Kapitelüberschrift „Wo das Einhorn zu finden.“ stellt er zunächst - eventuell implizit gegen Plinius‘ Erwähnung des Rhinoceros im Triumphzug des Pompeius gerichtet - das Vorkommen des Einhorns in Europa mit dem Argument in Frage, dass man es dort noch nie beobachtet habe: „NJemand ist/der dieses Thier jemahls in Europa gesehen habe: Und wiewol der Römische Pracht alle Thier der Erden in ihre Schauplätze und Triumpf zu bringen sich understanden lißt man doch nicht / daß das Einhorn jemahls dem Römischen Volck gezaiget worden sey/ da sonsten Tigerund Panterthiere/ Naßhörner/ Löwen/ Drachen/ etc und allerhand grausames Wild gefirrt und gezähmt/ vorgestellt worden.“40

Gesner zweifelt jedoch angesichts der überwältigenden Fülle an Zeugnissen nicht an der Existenz des Tieres und führt dafür auch handfeste Beweise an: „Deswegen man nur den Landfahrern unnd weitgereysten Leuten hiervon Glauben geben muß/ was sie sagen: Dann einmahl so ist das Thier auf Erden / sonst wären seine Hörner nicht vorhanden: Und läßt man es darbey bleiben / daß Indien / Arabien und Morenland sie erzeuge. Man saget auch daß es Wasser-Einhörner gebe / die sich im Wasser nähren/“41. Auch auf die Jagd mittels einer Jungfrau geht Gesner ein und verzeichnet eine Jagdlist, die auch die höfische Literatur hätte entwerfen können: Man verkleide einen starken Jüngling aus der Jagdgesellschaft als Jungfrau, der mit „wolriechendem Geruch balsamieret und angestrichen werde“" ${ }^{\text {42 }}$ und so das Einhorn täuschen soll: „Und wann das Thier den Geruch empfindet / und die Weibskleidung ersiehet / laufft es

36 Fratris Felicis Fabri, Evagatorium (wie Anm. 35), 441. Übersetzung von Klussmann, In Gottes Namen (wie Anm. 34), 98.

37 Ebd.

38 Conrad Gesner, Historiae animalium. Bd. 1: De quadrupedibus viviparis. Zürich 1551, „De monocerote“, 785-786; dt. Übersetzung: Conrad Gesner, Thierbuch, das ist ein kurtze beschreybung aller vierfüssigen Thiern, so auff der erden und in wassern wonend, übers. v. Conrad Forer. Zürich 1563, „Von dem Einhorn.", XXXVI-XXXXIX.

39 Hier zitiert nach der Ausgabe: Conrad Gesner, Allgemeines Thier-Buch: Abbildung aller vierfuessigen, sowohl zahmer als wilder Thieren, welche in allen vier Theilen der Welt auff dem Erdboden und in etlichen Wassern zu finden, sampt einer aussfuehrlichen Beschreibung. Unveränd. Nachdruck der Ausg. Frankfurt a. M.: Serlin 1669, Sonderausg. für J. F. Lehmanns Fachbuchhandlung. Hannover 1995, „Von dem Einhorn“, 71-83, hier 71.

40 Ebd., 76.

41 Ebd.

42 Ebd., 78. 
hinzu/ und legt sich dem Jungengesellen in den Schooß: Der dann mit seinen weiten und wolriechenden Ermeln das Thier blendet / bedeckt / und einschläffert: Alsdann lauffen die Jäger herbey / und nehmen das Horn vom Thier / das für Gifft gut ist/ das Einhorn aber lassen sie unverletzt wieder weglauffen. “43 Gesner selbst zweifelt zwar den Wahrheitsgehalt dieser Jagdgeschichte an, weist sie aber, seinem Universalanspruch folgend, auch nicht zurück: „Doch lassen wir solches in seynem Werth / und eines jeden Urtheil anheim gestellt." 44

Am Beginn der Frühmoderne hat man sich in Bezug auf das Einhorn also weder von den Denktraditionen der antiken Naturkunden noch von der Autorität der Bibel verabschiedet, wie auch schon an der Luther'schen Übersetzung des Tieres ,re'em' deutlich wurde. Gleichwohl spielen in der sich verwissenschaftlichenden Auseinandersetzung mit der Welt zunehmend Formen der empirischen Überprüfung und Autopsie eine Rolle: Einhörner muss es geben, denn schließlich werden ihre gedrechselten Hörner in den europäischen Wunderkammern und Kirchenschätzen in großer Zahl aufbewahrt und kommen auch in der Medizin, etwa bei der Behandlung von Vergiftungen zum Einsatz. Es handelte sich dabei natürlich um die Stoßzähne des Narwals, dem aus der Fachliteratur der Zeit auch als ,unicornu marinus ${ }^{\star 45}$ bekannten ,Wasser-Einhorn`. Bernd Roling hat in einem Beitrag zur Wissenschaftsgeschichte des 17. Jahrhunderts eindrücklich nachgezeichnet, wie das „Ende der Einhörner“ damit eingeläutet wurde, dass man die Heilkraft dieser Hörner zunehmend der experimentellen Überprüfung unterzog, u. a. indem man frühe Tierversuche unternahm, in denen Katzen, Tauben und Hunde zuerst mit Gift und dann als Gegenmittel mit geriebenem Einhorn traktiert wurden. ${ }^{46}$ Die pharmazeutische Wirkung - und damit ein für den Menschen zentraler Beziehungsaspekt - konnte dabei nicht umfassend widerlegt werden, so dass mit dem wissenschaftlichen Beweis, dass es sich bei den Hörnern um Narwalzähne handelte, zunächst lediglich „ihr Nimbus einfach auf das marine Einhorn übertragen “ ${ }^{47}$ wurde. Diese Erkenntnis führte aber, wie schon bei Gesner, nicht dazu, dass man den ,Landeinhörnern` grundsätzlich die Daseinsberechtigung aberkannte: „Gesichtet werden sie“ - allen Zweifeln an ihrer Existenz und an der Wirksamkeit der Hörner zum Trotz - „auch weiterhin“48, bis ins 19. Jahrhundert finden sich ihre Spuren in naturkundlichen Werken und Reiseberichten.

An der Schwelle zur Neuzeit beenden wir diese Spurensuche. Für ein „Tier das es nicht giebt“ ist das Einhorn erstaunlich präsent in der vormodernen (und unter veränderten Prämissen auch in der modernen) europäischen Vorstellungswelt. Unser Wissen um die Beschaffenheit der Welt und ihrer Bewohner, so wird an der Faszinations- und

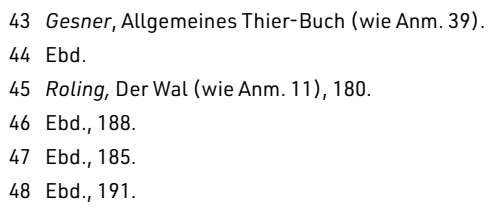


Wissensgeschichte des Einhorns deutlich, ist historisch gewachsen, und diese Historizität prägt unsere Wahrnehmung. Auch naturkundliche Fakten sind daher immer wieder darauf zu hinterfragen, aus welchen menschlichen Projektionen und Begehrensstrukturen heraus sie generiert werden. Die menschliche Sehnsucht nach der Nähe zum Einhorn jedenfalls scheint ungebrochen: Hätte die Meldung vom sibirischen Knochenfund überhaupt je Interesse gefunden, wenn sie „Lebten plumpe einhörnige Urzeitbisons mit dem Menschen zusammen?" gelautet hätte?

\section{Autorin}

\section{Julia Weitbrecht}

Professorin für Ältere deutsche Sprache und Literatur mit Schwerpunkt Literatur des Früh- und Hochmittelalters im europäischen Kontext an der Universität zu Köln. Ihre Forschungsschwerpunkte sind religiöse Textkulturen des Mittelalters, historische Narratologie und Mensch-Tier-Relationen in historischer Perspektive.

j.weitbrecht@uni-koeln-de 